PROCEEDINGS OF THE

AMERICAN MATHEMATICAL SOCIETY

Volume 126, Number 1, January 1998, Pages 189-194

S 0002-9939(98)04389-5

\title{
A THEOREM OF THE ALTERNATIVE IN BANACH LATTICES
}

JEAN B. LASSERRE

(Communicated by Palle E. T. Jorgensen)

\begin{abstract}
We consider a linear sytem in a Banach lattice and provide a simple theorem of the alternative (or Farkas lemma) without the usual closure condition.
\end{abstract}

\section{INTRODUCTION}

Let $X, Z$ be Banach spaces, with respective topological duals $X^{*}, Z^{*}$, and $A$ : $X^{*} \rightarrow Z^{*}$ a linear mapping. We consider the existence of solutions to the linear system $\{A y=b\}$ for some $b \in Z^{*}$ and when $X^{*}$ is a Banach lattice for some partial ordering $\leq$.

The usual way to get necessary and sufficient conditions is via a generalized Farkas Lemma (or Theorem of the Alternative) when $y$ is constrained to belong to some convex cone $S$ (the reader is referred to e.g. [3], [5] and the references therein). In [3] a crucial condition is the closure of $A(S)$ in some weak topology, rarely met in practice. In [5] one avoids this closure assumption via an augmented system when the space satisfies some topological assumptions.

We first treat the case where the convex cone $S$ is simply the whole space $X^{*}$ and therefore the closure condition on $A\left(X^{*}\right)$ is not satisfied. We assume that $I-A$ is not a compact operator, in which case the Fredholm alternative theorem answers the question.

In many applications of interest, $X^{*}$ has the structure of a Banach lattice for a natural partial ordering (e.g. the $L_{p}$ spaces for the Volterra and Fredholm type equations or the Poisson equation). We show that this particular structure permits us to derive a simple necessary and sufficient condition for existence of solutions. Incidently, this result also provides a necessary and sufficient condition for existence of a solution dominated by some pre-specified element $y_{0} \in X^{*}$. The result simplifies even more if $X^{*}$ has a unit vector $I$.

Finally, we also consider the existence of nonnegative solutions to $\{A y=b\}$, i.e. when the solution $y$ must be in the (convex) positive cone induced by the partial ordering. Examples of Banach lattices are also provided.

Received by the editors July 8, 1996.

1991 Mathematics Subject Classification. Primary 47A50, 46B42, 46H10.

Key words and phrases. Linear systems in Banach spaces, Farkas lemma, Banach lattices.

(C)1998 American Mathematical Society 


\section{NotATION AND DEFINITIONS}

Let $X$ be a separable Banach space with a partial ordering $\leq$ such that the positive cone $S$ (i.e. $x \leq y \Leftrightarrow y-x \in S$ ) is convex and strongly closed. $X$ is also assumed to be a vector lattice for this ordering.

Let $X^{*}$ be the topological dual of $X$ and $\leq$ the partial ordering on $X^{*}$ defined as $x \leq y \Leftrightarrow y-x \in S^{*}$, where $S^{*} \subset X^{*}$ is the dual cone of $S$.

$\left(X^{*}, \leq\right)$ is assumed to be a Banach lattice for this partial ordering, i.e. (i) $X^{*}$ is a vector lattice $(x \leq y \Rightarrow x+z \leq y+z)$, and (ii) if we denote by $|x|$ the absolute value of $x\left(|x|=(x) \vee(-x), x \in X^{*}\right)$, then

$$
|x| \leq|y| \Rightarrow\|x\| \leq\|y\|, \quad x, y \in X^{*} .
$$

Note that if $x^{+}\left(\operatorname{resp} . x^{-}\right)$is the usual notation for $x \vee 0$ (resp. $x \wedge 0$ ) then $|x|=x^{+}-x^{-}$.

Convergence in the weak* topology in $X^{*}$ is denoted by $\stackrel{w *}{\rightarrow}$.

Now, let $Z^{*}$ be the topological dual of a separable Banach space $Z$ (e.g. $Z:=X$ ), and $A: X^{*} \rightarrow Z^{*}$ a linear mapping. Both $\left(X^{*}, X\right)$ and $\left(Z^{*}, Z\right)$ form a dual pair of vector spaces (see e.g. [3]) and the adjoint $A^{*}: Z \rightarrow X$ is given by

$$
\left\langle y, A^{*} z\right\rangle=\langle A y, z\rangle \forall y \in X^{*}, z \in Z
$$

where $\langle.,$.$\rangle is the duality bracket for a dual pair. Convergence in Z^{*}$ for the weak* topology is also denoted by $t *$.

Consider the linear system:

$$
A y=b, y \in X^{*},
$$

for some $b \in Z^{*}$.

If $X^{*}$ is finite dimensional, then (2.2) has a solution if and only if

$$
\forall x \in Z, A^{*} x=0 \Rightarrow\langle b, x\rangle=0 .
$$

The same result (the Fredholm alternative) is true if $I-A$ is a compact operator $X^{*} \rightarrow X^{*}$.

If the solution to (2.2) is constrained to be in a convex cone $S^{*}$ (the dual cone of a strongly closed convex cone $S \subset X)$, then, if $A$ is continuous and $A\left(S^{*}\right)$ is closed (in the weak topologies induced by the dual pairs $\left(X^{*}, X\right)$ and $\left.\left(Z^{*}, Z\right)\right),(2.2)$ has a solution if and ony if

$$
\forall z \in Z, A^{*} z \in S \Rightarrow\langle b, z\rangle \geq 0 .
$$

This is the Generalized Farkas Theorem of Craven and Koliha, and the reader is referred to [3] for various results along these lines.

However, the condition $A\left(S^{*}\right)$ closed is crucial and rarely met in practice (even in finite dimensional spaces when the cone is not polyhedral). In [5], using an augmented system, we derived a Farkas lemma without this closure assumption.

The idea here is to also consider an augmented system, but we now use the Banach lattice property. That yields a particularly simple Farkas lemma.

\section{MAin RESUlts}

The system (2.2) has a solution $y \in X^{*}$ if and only if the system

$$
\left\{\begin{aligned}
A\left(y_{1}-y_{2}\right) & =b, \\
y_{1}+y_{2} & \leq y_{0}, \\
y_{1}, y_{2} & \in S^{*},
\end{aligned}\right.
$$


has a solution for some fixed $y_{0} \in X^{*}$ (note that, necessarily, $y_{0} \in S^{*}$ ).

Indeed, the if part is trivial, and if $y$ is a solution, then $\left(y_{1}, y_{2}\right)$ with $y_{1}:=y^{+}$ and $y_{2}:=-y^{-}$is a solution to the above system for any $y_{0} \geq|y|$.

As we show below, the above linear system (3.1) ensures the conditions that permit us to apply the Generalized Farkas Theorem of Craven and Koliha [3]. We begin with

Theorem 3.1. Let $A: X^{*} \rightarrow Z^{*}$ be such that $A^{*}(Z) \subseteq X$. Then the following two propositons are equivalent:

(a) $A y=b$ has a solution $y \in X^{*}$.

(b) For some $y_{0} \in S^{*},|\langle b, z\rangle| \leq\left\langle y_{0},\left|A^{*} z\right|\right\rangle \forall z \in Z$.

Proof. It suffices to consider (3.1). Consider the linear mapping $T:\left(X^{*}\right)^{3} \rightarrow$ $Z^{*} \times X^{*}$ with

$$
T\left(y_{1}, y_{2}, y_{3}\right)=\left[\begin{array}{c}
A y_{1}-A y_{2} \\
y_{1}+y_{2}+y_{3}
\end{array}\right]
$$

with adjoint $T^{*}: Z \times X \rightarrow X^{3}$ given by

$$
T^{*}(z, x)=\left[\begin{array}{c}
A^{*} z+x \\
-A^{*} z+x \\
x
\end{array}\right], z \in Z, x \in X .
$$

(3.1) has a solution if and only if

$$
T\left(y_{1}, y_{2}, y_{3}\right)=\left[\begin{array}{c}
b \\
y_{0}
\end{array}\right]
$$

has a solution $\left(y_{1}, y_{2}, y_{3}\right) \in\left(S^{*}\right)^{3}$.

We first prove that $T\left(S^{3}\right)$ is closed in the space $Z^{*} \times X^{*}$ for the weak topology $\sigma\left(Z^{*} \times X^{*}, Z \times X\right)$ induced by the dual pair $\left(Z^{*} \times X^{*}, Z \times X\right)$, i.e. for the weak* topology.

The weak* closure of a convex set in the dual of a separable Banach space can be characterized via converging sequences (see e.g. [4]).

Therefore, as $T\left(\left(S^{*}\right)^{3}\right) \subset Z^{*} \times X^{*}$ is convex, let $\left\{\left(y_{1}^{n}, y_{2}^{n}, y_{3}^{n}\right)\right\}$ be a sequence in $\left(S^{*}\right)^{3}$ such that $T\left(y_{1}^{n}, y_{2}^{n}, y_{3}^{n}\right)$ converges (weakly $\left.*\right)$ to some $\left(a_{1}, a_{2}\right) \in Z^{*} \times X^{*}$. We prove that $T\left(y_{1}, y_{2}, y_{3}\right)=\left(a_{1}, a_{2}\right)$ for some $\left(y_{1}, y_{2}, y_{3}\right) \in\left(S^{*}\right)^{3}$.

The weak convergence of $T\left(y_{1}^{n}, y_{2}^{n}, y_{3}^{n}\right)$ yields, in particular,

$$
y_{1}^{n}+y_{2}^{n}+y_{3}^{n} \stackrel{w *}{\rightarrow} a_{2}
$$

and

$$
A\left(y_{1}^{n}-y_{2}^{n}\right) \stackrel{w *}{\rightarrow} a_{1}
$$

Therefore (see e.g. [2]),

$$
\sup _{n}\left\|y_{1}^{n}+y_{2}^{n}+y_{3}^{n}\right\|<M
$$

for some $M>0$. Moreover, as $y_{i}^{n} \in S^{*},\left|y_{i}^{n}\right|=y_{i}^{n}$. In addition,

$$
\left|y_{i}^{n}\right| \leq\left|y_{1}^{n}+y_{2}^{n}+y_{3}^{n}\right| \forall i
$$

so that from (2.1) and (3.6) we have

$$
\sup _{n}\left\|y_{i}^{n}\right\| \leq \sup _{n}\left\|y_{1}^{n}+y_{2}^{n}+y_{3}^{n}\right\| \leq M, i=1,2,3 .
$$


As the unit ball in $X^{*}$ is weak* sequentially compact (see e.g. [2]), there is a subsequence $\left\{\left(y_{1}^{n_{k}}, y_{2}^{n_{k}}, y_{3}^{n_{k}}\right)\right\}$ such that

$$
y_{i}^{n_{k}} \stackrel{w *}{\rightarrow} y_{i}, \quad i=1,2,3 \text { as } k \rightarrow \infty .
$$

As $S^{*}$ is closed in the weak* topology, $y_{i} \in S^{*} \forall i$. In addition, as $A^{*}(Z) \subseteq X$, the mapping $A$ is weakly continuous for the $\left[\sigma\left(\left(X^{*}\right)^{3}, X^{3}\right), \sigma\left(Z^{*} \times X^{*}, Z \times X\right)\right]$ topologies, and thus $A\left(y_{1}^{n_{k}}-y_{2}^{n_{k}}\right) \stackrel{w *}{\rightarrow} A\left(y_{1}-y_{2}\right)$. Hence, from (3.5), $A\left(y_{1}-y_{2}\right)=a_{1}$.

Having proved that $T\left(\left(S^{*}\right)^{3}\right)$ is (weak*) closed, we can apply the Generalized Farkas Theorem of Craven and Koliha ([3], Theorem 2, p. 987, and Theorem 6, p. 989), according to which $T\left(y_{1}, y_{2}, y_{3}\right)=\left(b, y_{0}\right)$ has a solution in $\left(S^{*}\right)^{3}$ if and only if for every $(z, x) \in Z \times X$ that satisfy

$$
T^{*}(z, x) \in S^{3}
$$

then $\langle b, z\rangle+\left\langle y_{0}, x\right\rangle \geq 0$. Indeed, the dual cone in $X$ of $S^{*}$ is $\left(S^{*}\right)^{+}:=\{x \in$ $\left.X \mid\langle y, x\rangle \geq 0 \forall y \in S^{*}\right\}=S$, since $S$ is strongly closed (see e.g. [3]).

In view of (3.2), this yields: for every $(z, x) \in Z \times X$ with

$$
A^{*} z+x \in S,-A^{*} z+x \in S, x \in S,
$$

we have $\langle b, z\rangle+\left\langle y_{0}, x\right\rangle \geq 0$. As $y_{0} \in S^{*}$ and since we must have $x \geq A^{*} z$ and $x \geq-A^{*} z$, it suffices to check the above condition for $x:=A^{*} z \vee\left(-A^{*} z\right)$, i.e. $x:=\left|A^{*} z\right| \in S$, which yields $\langle b, z\rangle+\left\langle y_{0},\left|A^{*} z\right|\right\rangle \geq 0$. Now, the same condition for $-z$ yields $\langle b,-z\rangle+\left\langle y_{0},\left|A^{*} z\right|\right\rangle \geq 0$. Hence, we must have

$$
|\langle b, z\rangle| \leq\left\langle y_{0},\left|A^{*} z\right|\right\rangle \forall z \in Z,
$$

the desired result.

Therefore, checking whether (2.2) has a solution reduces to checking (b) for some $y_{0}$ that has to be guessed. Equivalently, checking whether (2.2) has no solution reduces to checking whether, for every $y_{0} \in S^{*},|\langle b, z\rangle|>\left\langle y_{0},\left|A^{*} z\right|\right\rangle$ for some $z \in Z$.

Incidently, for a given $y_{0} \in S^{*}$, (b) also gives a necesary and sufficient condition for the existence of a solution $y$ such that $|y|$ is dominated by $y_{0}$, which is of interest in some applications.

An important particular case is when the Banach lattice $X^{*}$ has a unit vector $I$ with the property (see e.g. [6])

$$
I>0 \text { and } \forall y \in X^{*}, \exists \alpha>0 \text { such that }-\alpha I \leq y \leq \alpha I .
$$

In this case we can use $y_{0}:=\gamma I$ in (3.1) to obtain, finally,

Corollary 3.2. Let $A: X^{*} \rightarrow Z^{*}$ be such that $A^{*}(Z) \subseteq X$. Then the following two propositons are equivalent:

(a) $A y=b$ has a solution $y \in X^{*}$.

(b) For some $\gamma>0,|\langle b, z\rangle| \leq \gamma\left\langle I,\left|A^{*} z\right|\right\rangle \forall z \in Z$.

\section{Cone constrained linear System}

We now consider the same linear system $\{A y=b\}$, but we now require the solution $y$ to be in $S^{*}$, i.e. one requires a nonnegative solution. In this case we get: 
Theorem 4.1. Let $A: X^{*} \rightarrow Z^{*}$ be such that $A^{*}(Z) \subseteq X$. Then the following two propositons are equivalent:

(a) $A y=b$ has a solution $y \in S^{*}$.

(b) For some $y_{0} \in S^{*},\left\langle y_{0},\left(A^{*} z\right)^{-}\right\rangle \leq\langle b, z\rangle \leq\left\langle y_{0},\left(A^{*} z\right)^{+}\right\rangle \forall z \in Z$.

In addition, if $X^{*}$ has a unit vector $I$, then $(b)$ becomes

$\left(b^{\prime}\right)$ For some $\gamma>0, \gamma\left\langle I,\left(A^{*} z\right)^{-}\right\rangle \leq\langle b, z\rangle \leq \gamma\left\langle I,\left(A^{*} z\right)^{+}\right\rangle \forall z \in Z$.

Proof. The proof is along the same lines as for Theorem 3.1, except now we consider the linear mapping $T:\left(X^{*}\right)^{2} \rightarrow Z^{*} \times X^{*}$ defined as:

$$
T\left(y_{1}, y_{2}\right)=\left[\begin{array}{c}
A y_{1} \\
y_{1}+y_{2}
\end{array}\right] .
$$

Indeed, the system $\{A y=b\}$ has a solution $y \in S^{*}$ if and only if $\{A y=b, 0 \leq y \leq$ $\left.y_{0}\right\}$ has a solution for some $y_{0} \in S^{*}$. As in the proof of Theorem 3.1 we can prove that $T\left(\left(S^{*}\right)^{2}\right)$ is (weak*) closed, so that again we can apply the Generalized Farkas Theorem of Craven and Koliha [3], which yields

$$
z \in Z, x \in S, A^{*} z+x \in S \Rightarrow\langle b, z\rangle+\left\langle y_{0}, x\right\rangle \geq 0 .
$$

In (4.1), since $x \geq-A^{*} z$ and $x \in S$, it suffices to consider $x:=\left(-A^{*} z\right) \vee 0=$ $-\left(A^{*} z\right)^{-}$, which yields $\langle b, z\rangle \geq\left\langle y_{0},\left(A^{*} z\right)^{-}\right\rangle$. A similar argument with $-z$ yields $\langle b, z\rangle \leq\left\langle y_{0},\left(A^{*} z\right)^{+}\right\rangle$, i.e. (b).

If $X^{*}$ has a unit vector $I$, then just replace $y_{0}$ by $\gamma I$ for some scalar $\gamma>0$.

Examples. Below is a list of some Banach lattices.

- $X:=R^{n(n+1) / 2}=: X^{*}$, identified with the space of real-symmetric $(n, n)$ matrices with the partial ordering $x \leq y \Leftrightarrow y-x$ positive semi-definite. In this case, $X^{*}$ has finite dimension but the cone $S^{*}$ is not polyhedral, so that the standard Farkas lemma does not apply. The identity matrix $I$ is a unit vector.

- $X^{*}:=L^{p}(\Omega, \mathcal{B}, \mu)$ (with $\left.1<p \leq \infty\right)$ is a Banach lattice for the natural partial ordering $x \leq y \Leftrightarrow x(\omega) \leq y(\omega), \omega \in \Omega$.

- $X^{*}:=l_{p}$ (with $1 \leq p \leq \infty$ ). When $X^{*}:=l_{1}$ then $X:=c_{0}$, the space of sequences that vanish at infinity.

- $X:=C_{0}(\Omega)$, the space of continuous functions on $\Omega$ that vanish at infinity (with $\Omega$ a separable locally compact Hausdorff space). Then $X^{*}:=M(\Omega)$, the space of finite Borel signed measures on $(\Omega, \mathcal{B})$, and the partial ordering $\mu \leq \nu$ is also the natural ordering $\mu(B) \leq \nu(B), B \in \mathcal{B}$.

For examples of particular applications of interest, let us just mention Lyapunov and Riccati equations (or inequalities) for positive semi-definite matrices, Volterra and Fredholm type equations in $L_{p}$ spaces, as well as the Poisson equation.

\section{REFERENCES}

1. R.B. Ash, Real Analysis and Probability, Academic Press, New York, 1972. MR 55:8280

2. H. Brézis, Analyse Fonctionnelle, Masson, Paris, 1983 MR 85a:46001

3. B.D. Craven and J.J. Koliha, Generalizations of Farkas' Theorem, SIAM J. Math. Anal. 8 (1977), 983-997. MR 57:11039

4. N. Dunford, J.T. Schwartz, Linear Operators. Part I: General Theory, Interscience Publishers, Inc., New York, 1958. MR 22:8302 
5. J.B. Lasserre, A Farkas Lemma without a standard closure condition, SIAM J. Contr. Optim. 35 (1997), 265-272. CMP 97:07

6. K. Yosida, Functional Analysis, 6th ed., Springer-Verlag, New York, 1980. MR 82i:46002

LAAS-CNRS, 7 Av. du Colonel Roche, 31077 Toulouse Cédex, France

E-mail address: lasserre@laas.fr 